\title{
Monitoring of Brain Hemodynamics Coupling in Neonates using Updated Tensor Decompositions
}

\author{
A Caicedo, O De Wel, M Vandecappelle, L Thewissen, A Smits, K Allegaert, L. De Lathauwer, G \\ Naulaers, and S Van Huffel.
}

\begin{abstract}
In this paper we explore the use of updated tensor decompositions for the monitoring of brain hemodynamics in neonates. For this study, we used concomitant measurements of heart rate, mean arterial blood pressure, arterial oxygen saturation, EEG, and brain oxygenation - measured using nearinfrared spectroscopy. These measurements were obtained from 22 neonates undergoing an INSURE procedure (INtubation, SURfactant and Extubation) and sedation using propofol. To develop the monitoring framework using tensors, we used radial basis kernel function (RBF) to construct a similarity matrix for consecutive segments of the signals. These matrices were concatenated forming a tensor. Updating canonical polyadic decomposition was used to evaluate the impact of propofol in the coupling between the different signals. Results indicate, as previously reported, a drop in the interaction between signals due to propofol administration. This shows that tensor decompositions can be useful in order to monitor the coupling between different physiological signals.
\end{abstract}

\section{INTRODUCTION}

Tensors are mathematical objects that generalize the concepts of vectors and matrices. Recently tensors have gained popularity due to their versatility, and because some problems are easily represented using these types of structure [1]. For instance, in clinical applications such as monitoring, tensors are attractive since the data can naturally be organized in a tensor. Moreover, tensor decomposition methods allow to store the information in a compact way, enabling the analysis of large datasets, such as the ones obtained from medical devices.

Normally, monitoring systems record different modalities simultaneously and visualize these on one screen. By analyzing the common dynamics of these signals, it is possible to infer the status of the patient. However, due to the nonstationary characteristics of human physiology, the interaction between these signals is likely to change in time. This type of changes can be tracked using tensors. Nevertheless, since monitoring devices are always acquiring new data, the storage of the information in the tensor structure can be challenging; therefore, updating tensor decomposition techniques should be considered. These techniques do not

\footnotetext{
*The research leading to these results has received funding from the European Research Council under the European Union's Seventh Framework Programme (FP7/2007-2013) / ERC Advanced Grant: BIOTENSORS (no. 339804). This paper reflects only the authors' views and the Union is not liable for any use that may be made of the contained information.

A.C, O.D.W and S.V.H (e-mail: sabine.vanhuffel@esat.kuleuven.be) are with the Department of Electrical Engineering (ESAT), STADIUS Center for Dynamical Systems, Signal Processing and Data Analytics, KU Leuven, Belgium; and with imec, Leuven, Belgium.

A.C. is also with the Department of Applied Mathematics and Computer Sciences, Universidad del Rosario, Bogotá, Colombia.
}

process all the data at once, but sequentially, to update the tensor decomposition. For instance, in [2] a canonical polyadic decomposition (CPD) updating algorithm is proposed. This algorithm sequentially updates the decomposition of a tensor using as input the CPD of the initial tensor. New slices corresponding to the new measurements are added sequentially to update the CPD, thereby exploiting the CPD structure of the data tensor as much as possible.

In this study, we use CPD updating for the monitoring of brain hemodynamics in neonates. More specifically we investigate the effect of the administration of propofol in neonates. Propofol is an anesthetic that is used in neonates. It has a rapid onset and a short duration. This model has been chosen because we have previously reported in several studies the effects of propofol on the neonates [3, 4]. We have observed that as side effects propofol induces a decrease in mean arterial blood pressure (MABP) [4], as well as a decoupling of the common dynamics between different signals [5], and a reduction in the aEEG background pattern during the first hour after propofol administration [6]. We use the results from these previous studies as a reference to compare the results provided by the CPD updating.

\section{METHODS}

\section{A. Data}

The dataset consists of measurements from 22 neonates undergoing an INSURE (INtubation, SURfactant and Extubation) procedure. It contains concomitant measurements of heart rate (HR), MABP, arterial oxygen saturation $\left(\mathrm{SaO}_{2}\right)$, EEG, and brain oxygenation $\left(\mathrm{rScO}_{2}\right)$ - measured by means of Near-InfraRed Spectroscopy (NIRS). The data for this study is a subsample from the data reported in [3]. In [3], 50 neonates were sedated using propofol as part of an endothracheal intubation procedure. The complete dataset was recorded at the Neonatal Intensive Care Unit of the University Hospitals Leuven, Gasthuisberg. Ethical approval was provided from the same institution. The trial was registered on ClinicalTrials.gov NCT01624373. From the 50 subjects, data from only 22 subjects could be used for this study, due to the quality and

L.T, A.S. and G.N. are with the Department of Development and Regeneration, Neonatal Intensive Care Unit, UZ Leuven, Belgium

M.V. and L.D.L. are with ESAT-Stadius, EE Department, KU Leuven, Belgium and Group Science, Engineering and Technology, KU Leuven Kulak, Kortrijk, Belgium.

KA is with the Department of Development and Regeneration, KU Leuven, Belgium., and with the Department of Pediatrics, Division of Neonatology, Erasmus MC Sophia Children's Hospital, University Medical Center Rotterdam, the Netherlands. 

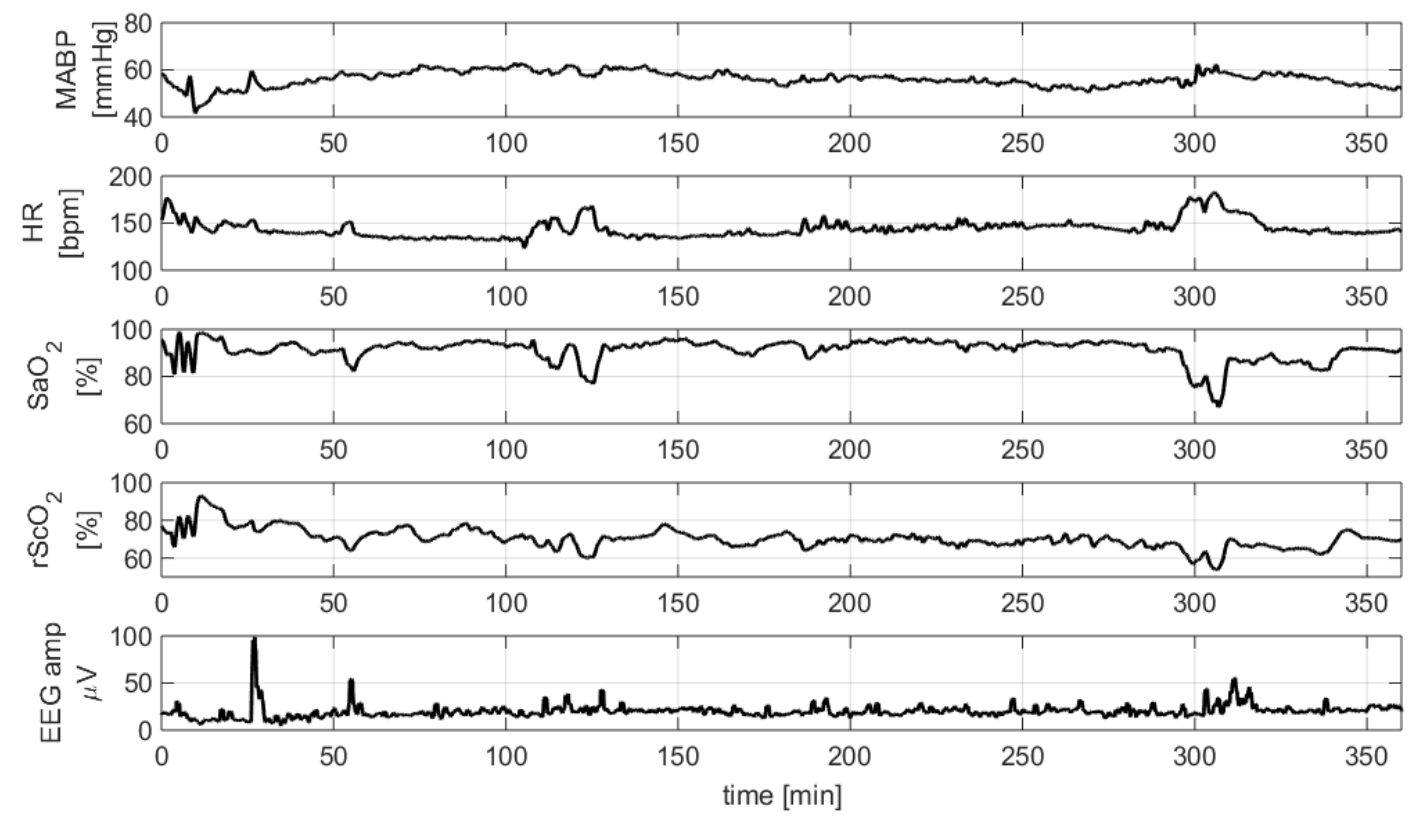

Figure 1. Concomitant measurements from a characteristic neonate after propofol administration. The signals are shown after the preprocessing step.

availability of the measurements. As part of the study protocol, all 22 included neonates underwent sedation using propofol, and the measurements were recorded up to 10 hours after propofol administration. The postmenstrual age (PMA) of the neonates expressed in median (range) was 30 (26-35) weeks, and the dose of propofol was $1.0(0.5-4.5) \mathrm{mg} / \mathrm{kg}$. The data was divided in 3 different groups based on the PMA as follows: extremely preterm (5 neonates) with a PMA $<28$ weeks, very preterm (13 neonates) 28 weeks $<$ PMA $<316 / 7$ weeks, and moderate to late preterm (4 neonates) 32 weeks $<$ PMA $<36$ 6/7 weeks. The HR, MABP, and $\mathrm{SaO}_{2}$ were measured with IntelliVue MP70 (Philips, Eindhoven, The Netherlands), the NIRS signal was measured with INVOS 5100 (Covidien, Mansfield, Massachusetts), and the EEG was measured between the C3 and C4 electrodes, according to the 10-20 system with a cerebral function monitor (Olympic CFM 6000, Natus). More information about this dataset is reported in [3, 5].

\section{B. Preprocessing}

The MABP, $\mathrm{HR}, \mathrm{SaO}_{2}$, and $\mathrm{rScO}_{2}$ were recorded with a sampling frequency $(F s)$ of $1 \mathrm{~Hz}$, while the EEG signal was recorded using a $F s=100 \mathrm{~Hz}$. The signals were preprocessed by computing their root mean square (RMS) value in a sliding window of one-minute having an overlap of 59 seconds. Therefore a new RMS value is provided every second. We used the same preprocessing scheme for the EEG. In this way the surrogate signal representing the EEG activity will have the same sampling frequency as the other signals. From each neonate we analyzed 6 hours of data after the administration of propofol, since it is assumed that by that time the effect of propofol in the neonates is negligible [3, 4]. In figure 1 measurements, after preprocessing, from a representative subject are shown.

\section{Algorithm}

In order to simulate a real monitoring scheme, we divided the available data in consecutive segments of 5 minutes, having an overlap of 4 minutes and 50 seconds, i.e. every 10 seconds we process the last 5 minutes of data. The similarity between the different signals was computed using an RBF kernel as follows: $(i, j)=e^{-\frac{\left\|x_{i}-x_{j}\right\|_{2}^{2}}{\sigma^{2}}}$, where $S(i, j)$ represents the similarity measurements between the signals $\boldsymbol{x}_{i}$ and $\boldsymbol{x}_{j}$, and $\sigma^{2}$ is the kernel bandwidth. After computing the similarity measurement from each segment of the recorded signals, we obtain a $5 \times 5$ matrix. In order to find an adequate parameter for $\sigma^{2}$ in the RBF kernel function, we concatenated all kernel matrices $S$, obtained from the different signal segments and all subjects. We computed the $\sigma^{2}$ value maximizing the Shannon entropy of the resulting enlarged matrix, and used it as a fixed parameter for further analysis. By computing the kernel bandwidth in this way, we guarantee maximum excursion in the values computed with the RBF kernel.

To initialize the algorithm we used the first 10 minutes of data, which contain 31 different segments, producing 31 5x5 similarity matrices. These matrices are stacked as frontal slices of a third order tensor, forming an initial tensor structure of dimensions $5 \times 5 \times 31$. Since the similarity matrices are symmetric, the first and second mode of the resulting tensor represent the same information, the similarity between one signal modality and the others. The third mode in the tensor represents the changes in time of the similarity measurements among the signals, and it is used as a monitoring variable. We used a rank-one CPD in order to retrieve information about the changes in the similarity measurement among the different signals, represented in the third mode of the decomposed tensor. New data is added to the tensor as new slices in its third mode. To avoid the tensor growing in this way and taking into account the limited 
computational resources, we used tensor decomposition updating proposed in [2]. This algorithm only requires the matrices obtained from the CPD of the initial tensor. These matrices initialize the decomposition as specified in [2]. After updating the decomposition of the new enlarged tensor, we obtain the updated third mode, which is used as a monitoring variable, and represents the changes in the similarity measurements between the different signals.

In summary, the monitoring algorithm consists of the following steps:

1. Computing the RMS value of the signals using a sliding one-minute window.

2. Segmentation of the data using five-minute windows, with an overlap of 4 minutes and 50 seconds.

3. Computation of the similarity matrices using the RBF kernel, and construction of the initial tensor structure, using the first 10 minutes.

4. CPD rank-one of the initial tensor.

5. Generation of the new slices of the tensor using the available data from the next 10 seconds.

6. Computation of the CPD updating, as specified in [2].

7. The algorithm repeats as long as new data is acquired.

\section{RESUlTS}

In figure 2, the factor in the third mode of the tensor decomposition for all the subjects are shown. The results are presented as the median value (black solid line), and the 25 and 75 percentile (shadow area). In order to detect the return to baseline level we performed a statistical analysis using the Kruskal-Wallis test on the time series obtained from the median value (black solid line in Figure 2). For the test the data was segmented in 2 parts, an initial part until a time t, and the rest of the time series. We identify the return to baseline level as the time $t$ when the 2 data segments were no longer statistically different $(\mathrm{p}>0.05)$. For the complete dataset we obtained a return to baseline level in 59 minutes. This time is indicated in Figure 2 as the dashed line.

In figure 3, the factor in the third mode of the tensor decomposition per patient group is depicted. From left to right, the results show the third mode for the extremely premature, very premature and the late premature neonates. The dashed line indicates the stabilization time, for each group, as presented in figure 2 . We performed the same statistical analysis to identify the stabilization times in the three different groups and we found a stabilization time of 114, 26 and 28 minutes for the extremely premature, very premature and late premature population. The figure shows that the results from the extremely premature children present a larger value in magnitude, as well as a larger variability. It also suggests that the stabilization time for the extremely premature neonates is larger than the one computed for the complete patient group. The results for the very premature and late premature neonates are more similar in magnitude and present a lower variability between the subjects. But, their stabilization time is smaller than the stabilization time computed from the complete population.

We also studied the relationship between the dose of propofol and the depth of the drop in the coupling between the variables, indicated by the third mode of the tensor

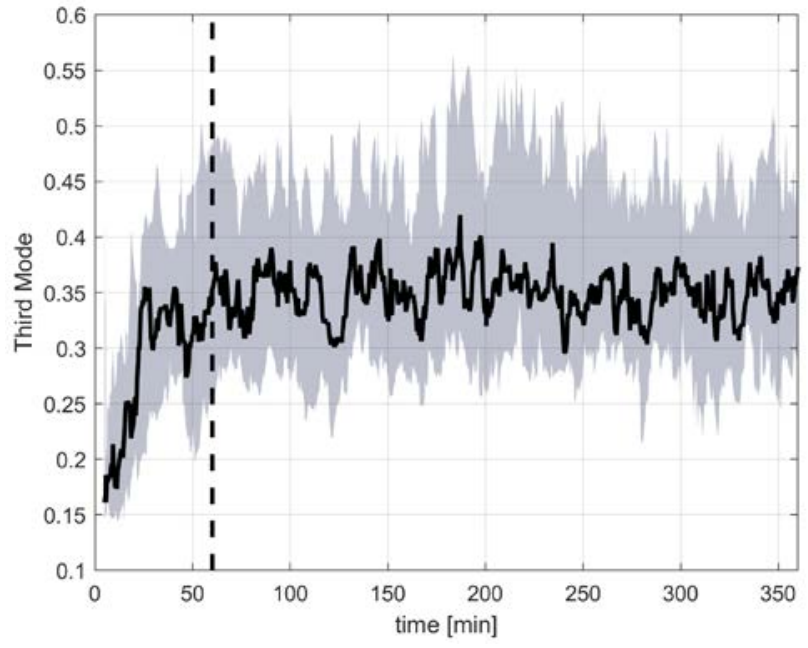

Figure 2. Third mode of the decomposed tensor represented as the median value (black solid line), and the 25 and 75 percentile (shadow area). This mode indicates the changes in time of the interaction among the signals. The dashed line represents the time when the signal coupling returns to the baseline level.

decomposition. However, no relation was found between these variables. This is not unexpected, since no pharmacokinetics data was included in this study.

\section{DISCUSSION}

We have shown that CPD updating can be used in order to track the changes in the common dynamics between different signal modalities. By using a similarity matrix, obtained by means of an RBF function, it is possible to estimate the coupling between different time series. Changes in the coupling between these variables can be tracked by stacking the similarity matrix from different time segments, and using an appropriate tensor decomposition. Since the aim is to use this methodology for real-time monitoring, the tensor decomposition needs to be updated.

The results obtained using updating of the tensor decomposition indicate that the common dynamics of the signals are disrupted during the first hour after propofol administration. Previous results reported by our group have shown a stabilization time of 90 minutes [5]. Even though these results suggest a smaller stabilization time they are in agreement with clinical results presented in [2, 3, 7], where a drop in MABP was observed within the first hour after propofol administration. Since the drop in MABP is one of the main contributions to the decoupling between the different signal modalities, as shown in [5], the results provided by CPD updating might reflect this.

Additionally, different dynamics in the third mode of the tensor decomposition were observed among different patient groups. The extremely premature group PMA $<28$ weeks presents not only a higher magnitude and variability in the monitoring variable, but also seems to present a slower return to baseline level (114 minutes). Moreover, the very preterm and late preterm groups exhibits a faster recovery time, 26 and 28 minutes respectively. These results are similar to the ones presented in [5]. However, in [5] the extremely premature subjects exhibit a behavior closer to the one reported for very premature subjects, whilst in this study their behavior was 

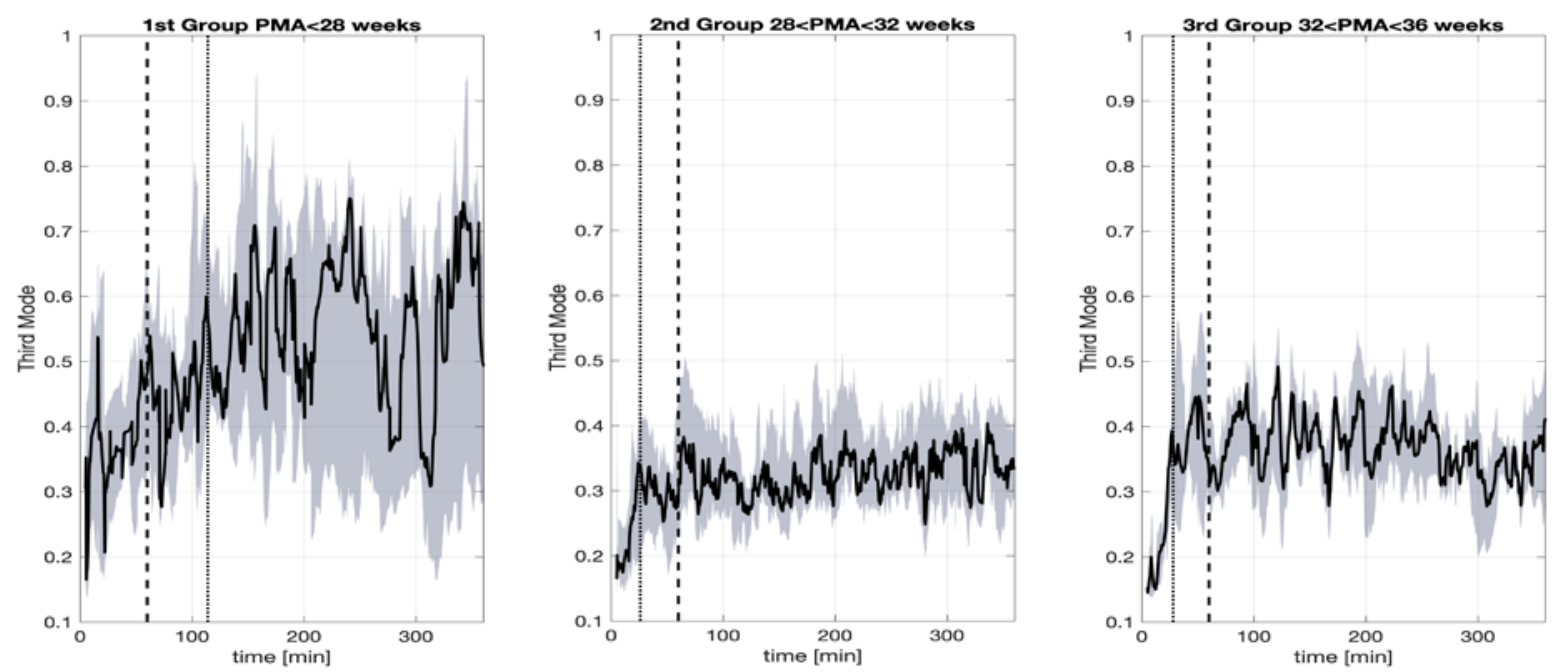

Figure 3. Third mode of the decomposed tensor for the three different groups. The dashed line represents the stabilization time for the complete dataset, while the dotted line represents the stabilization time for each group.

closer to the oldest group. Whether this is a maturational effect or PK driven effect needs further analysis.

We also analyzed the changes produced in the first and second mode during the updating. However, they do not seem to vary in a specific pattern. We hypothesize that these changes reflect the relation between one variable and the others; however, further research in the interpretation of these modes is needed. Due to space limitations we could not include a figure of these changes.

It is important to notice that in [5] a dynamic graph was used in order to monitor the coupling between different signal modalities, and assess the changes in their dynamics by means of graph metrics. These dynamic graphs can be related to tensor decompositions by studying these as a multi-way structure of a time-evolving network, as shown in [8].

One important drawback of this study is the lack of reference values prior to propofol administration. Since at least 5 minutes of data are required in order to produce a baseline value, it is difficult in this kind of procedures to obtain these data. However, it has been reported that the effects of propofol after 6 hours are negligible [5]. For this reason, we analyze only the first 6 hours after propofol administration.

\section{CONCLUSION}

We have shown that CPD tensor updating can be used for monitoring the coupling between different signal modalities. The results obtained in this study are similar to the results reported previously by our group, where it was found that propofol induces a disruption of the coupling between the common dynamics of the different signals. In addition, these results suggest also a link between tensor decompositions and dynamic graphs. This link has been previously reported in the literature for the analysis of social networks [8], but here we show their relation in the field of network physiology.

\section{ACKNOWLEDGMENT}

This research is supported by Bijzonder Onderzoeksfonds (BOF), KU Leuven: the effect of perinatal stress on the later outcome in preterm babies (no. C24/15/036); Fonds voor Wetenschappelijk Onderzoek (FWO), Vlaanderen: Hercules Foundation (AKUL 043) "Flanders BCI Lab-High-End, Modular EEG Equipment for Brain Computer Interfacing”; imec funds 2017. M.V. is supported by a PhD SB grant from FWO.

\section{REFERENCES}

[1] A. Cichocki, C. Mandic, A.H. Phan, C. Caiafa, G. Zhou, Q. Zhao, and L. De Lathauwer, "Tensor decompositions for signal processing applications. From two-way to multiway component analysis," IEEE Signal Processing Magazine, vol. 32, pp. 145-163, 2015.

[2] M. Vandecappelle, N. Vervliet, and L. De Lathauwer. "Nonlinear least squares updating of the canonical polyadic decomposition." Signal Processing Conference (EUSIPCO), 2017 25th European. IEEE, 2017.

[3] A. Smits, L. Thewissen, A. Caicedo, G. Naulaers, and K. Allegaert, "Propofol dose-finding to reach optimal effect for (semi-)elective intubation in neonates," The Journal of Pediatrics, vol. 179, pp. 5460.e9, 2016.

[4] J. Vanderhaegen, G. Naulaers, S. Van Huffel, C. Vanhole, and K. Allegaert, "Cerebral and systemic hemodynamic effects of intravenous bolus administration of propofol in neonates”, Neonatology, vol. 98, no. 1, pp. 57-63, 2010.

[5] D. Hendrikx, L. Thewissen, A. Smits, G. Naulaers, K. Allegaert, S. Van Huffel, and A. Caicedo, "Using Graph Theory to Assess the Interaction between Cerebral Function, Brain Hemodynamics, and Systemic Variables in Premature Infants”. Complexity. Article ID 6504039, 2018.

[6] Thewissen, L., Caicedo, A., Dereymaeker, A., Van Huffel, S., Naulaers, G., Allegaert, K., \& Smits, A. (2018). Cerebral autoregulation and activity after propofol for endotracheal intubation in preterm neonates. Pediatric research, 84(5), 719.

[7] S. Ghanta, M. E. Abdel-Latif, K. Lui, H. Ravindranathan, J. Awad, and J. Oei, "Propofol compared with the morphine, atropine and suxamethonium regimen as induction agents for neonatal endotracheal intubation: a randomized, controlled trial”, Pediatrics, vol. 119, no. 6, pp. e1248-e1255, 2007.

[8] S. Fernandes, H. Fanaee-T, \& J. Gama, "Dynamic graph summarization: a tensor decomposition approach”. Data Mining and Knowledge Discovery, 32(5), 1397-1420, 2018. 